\title{
Hydronephrosis: an unusual complication of neurogenic bowel in a patient with spinal cord injury: a case report
}

\author{
LP Lai ${ }^{1}$, CM Gibson-Gill ${ }^{1,2}$, G Gombas ${ }^{2}, \mathrm{~K}_{\text {Claffey }}^{2}$, S Tanks ${ }^{2}$ and S Garstang ${ }^{1,2}$
}

Study design/methods: Case report.

Objectives: In this case report, a consequence of not following proper care of the bowel affecting the genitourinary system is reported and discussed.

Setting: United States.

Results/conclusion: Neurogenic bowel and bladder can result from a spinal cord injury. It is necessary for spinal cord injury patients to continually follow recommended bladder and bowel care programs to decrease complications.

Spinal Cord (2014) 52, S9-S10; doi:10.1038/sc.2014.55

\section{INTRODUCTION}

The incidence of spinal cord injury is relatively low compared with other chronic neurologic impairments such as stroke (183 per 100000 versus 4.5 per 100000$).{ }^{1}$ However, the sequelae can have devastating effects to the patient and the family. The economic impact is also high. A study conducted in the Veteran Health Administration revealed a cost of $\$ 21450$ per patient in the fiscal year of $2005 .^{2}$

Neurogenic bowel and bladder can result from a spinal cord injury. It is necessary for spinal cord injury patients to continually follow recommended bladder and bowel care programs to decrease complications. In this case report, a consequence of not following proper care of the bowel affecting the genitourinary system is reported and discussed.

\section{CASE REPORT}

A 67-year-old male veteran, with a T2 American Spinal Injury Association scale A spinal cord injury after a motor vehicle accident resulting in paraplegia, neurogenic bladder and bowel presented to the Emergency Department with fevers and weakness. At home he was self-catheterizing about every $4 \mathrm{~h}$ to manage his neurogenic bladder and occasionally taking stool softeners for his neurogenic bowel. He reported that he had some difficulty with bowel care recently and noticed that he had also developed occasional urinary leakage between his intermittent catheterization times. Urinalysis revealed negative leukocyte esterase, 5-8 white blood cells and negative nitrites. He was admitted for a presumed urinary tract infection and started on intravenous antibiotics. During admission, the patient again developed fever and chills so a workup was repeated. Laboratory findings were essentially normal except for an elevated alkaline phosphatase.

Computed tomography (CT) of the abdomen and pelvis was performed to look for a possible source and it revealed a new mild-tomoderate hydronephrosis at the level of the left ureteropelvic junction with no evidence of radiopaque renal calculi, and severe constipation throughout the colon (Figure 1). Stool softeners, a bowel stimulant and daily bowel care performed by the spinal cord injury nursing staff were prescribed with good results. A follow-up ultrasound performed a week after the CT scan revealed no hydronephrosis (Figure 2). The patient had no further fevers or chills.

\section{DISCUSSION}

Regimented bowel care is imperative in patients with spinal cord injuries who have neurogenic bowel. In this case, the stool impaction most likely resulted in the new onset left hydronephrosis. There have been previously reported cases of obstipation causing hydronephrosis in an adolescent and a toddler. ${ }^{3}$ However, no cases have been reported in a patient with spinal cord injury who has a neurogenic bowel.

It is known in the obstetrics and gynecological literature of high incidence of hydronephrosis during pregnancy. One etiology is the mechanical compression of the ureters by the pregnant womb at the level of the pelvic brim. ${ }^{4-6}$ This can be applied to our case in which the impacted stool and colonic distension may mechanically compress the ureters.

Given the serious nature and the sequelae of hydronephrosis, it is necessary to recognize that the cause of hydronephrosis in the spinal cord-injured patient could very well be obstipation. Furthermore, after the removal of the stool, a discussion to follow proper bowel care recommendations with the patient and/or caregiver should be accomplished to prevent future hydronephrosis.

\section{CONCLUSIONS}

When considering etiologies of hydronephrosis in a patient with spinal cord injury and a neurogenic bowel, improper bowel emptying should be considered. A CT scan of the pelvis and abdomen may elucidate the cause of the hydronephrosis and guide further

${ }^{1}$ Department of Physical Medicine and Rehabilitation, Rutgers New Jersey Medical School, Newark, NJ, USA and ${ }^{2}$ Veterans Affairs New Jersey Health Care System, East Orange, NJ, USA

Correspondence: CM Gibson-Gill, Veterans Affairs New Jersey Health Care System, East Orange, NJ 07018, USA.

E-mail: lawrencelai1998@yahoo.com

Received 11 February 2014; revised 5 March 2014; accepted 16 March 2014 


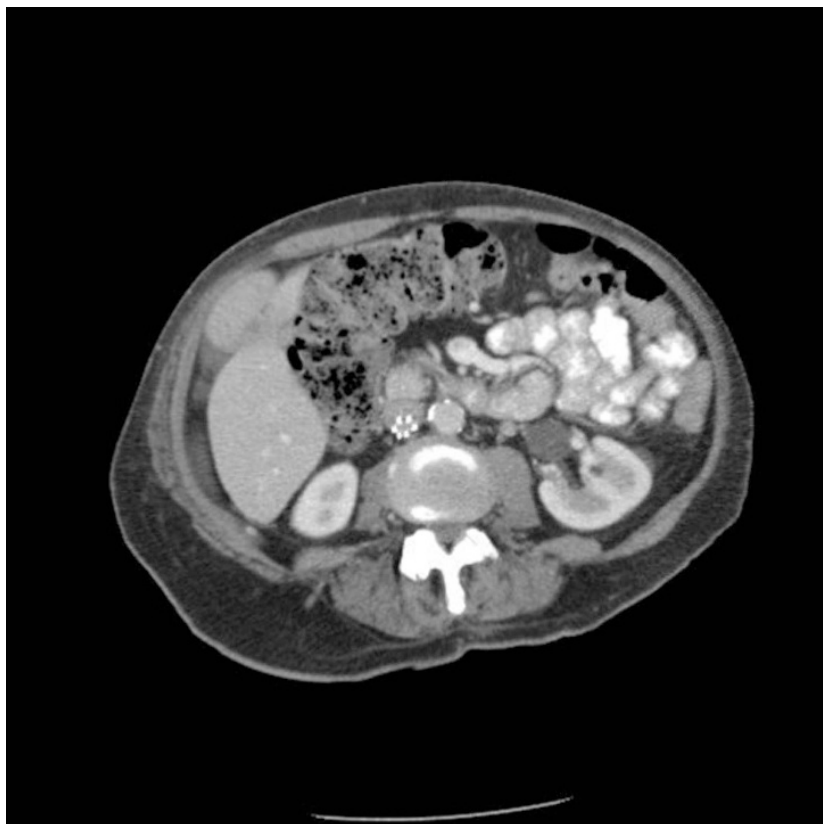

Figure $1 \mathrm{CT}$ scan of the abdomen and pelvis demonstrating mild-tomoderate hydronephrosis at the level of the left ureteropelvic junction.

management. The importance of proper management of neurogenic bowel should be emphasized to both patients and caregivers.

\section{CONFLICT OF INTEREST}

The authors declare no conflict of interest.

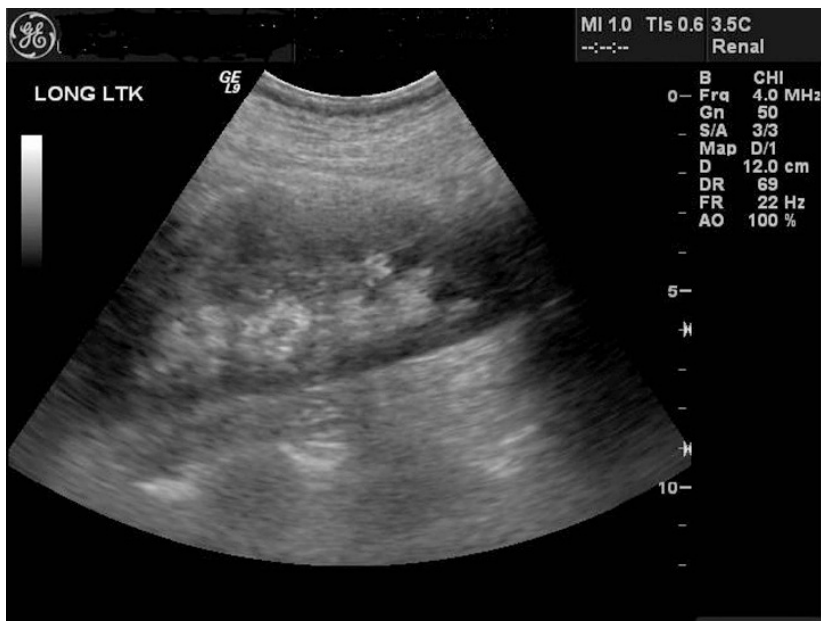

Figure 2 Follow-up ultrasound demonstrating no evidence of hydronephrosis.

1 Hirtz D, Thurman DJ, Gwinn-Hardy K, Mohamed M, Chaudhuri AR, Zalutsky R. How common are the "common" neurologic disorders? Neurology 2007; 68: 326-337.

2 French DD, Campbell RR, Sabharwal S, Nelson AL, Palacios PA, Gavin-Dreschnack DJ. Health care costs for patients with chronic spinal cord injury in the Veterans Health Administration. Spinal Cord Med 2007; 30: 477-481.

3 Ruoss KA, O'Sullivan R. Chronic constipation causing obstructive uropathy in an adolescent male. Pediatr Emerg Care 2008; 24: 462-463.

4 Au KK, Woo JS, Tang LC, Liang ST. Aetiological factors in the genesis of pregnancy hydronephrosis. Aust N Z J Obstet Gynaecol 1985; 25: 248-251.

5 Woo JS, Wan CW, Ma HK. Pregnancy hydronephrosis-a longitudinal ultrasonic evaluation. Aust N Z J Obstet Gynaecol 1984; 24: 9-13.

6 Leyendecker JR, Gorengaut V, Brown JJ. MR imaging of maternal diseases of the abdomen and pelvis during pregnancy and the immediate postpartum period. Radiographics 2004; 24: 1301-1316. 